\title{
Análisis de trade-off en el borde de una reserva forestal en el gradiente urbano-rural de Bogotá
}

Trade-off Analysis on a Forest Reserve Edge in Bogota's Rural-Urban Gradient

Análise de trade-offs na beira de uma reserva florestal no gradiente urbano-rural de Bogotá

\author{
Elcy Corrales Roa*
}

Viviana Osorno Acosta ${ }^{*}$

Recibido: 6 de julio de 2017

Aprobado: 3 de octubre de 2017

Doi: http://dx.doi.org/10.12804/revistas.urosario.edu.co/territorios/a.5909

Para citar este artículo:

Corrales, E. \& Osorno, V. (2018). Análisis de trade-off en el borde de una reserva forestal en el gradiente urbano-rural de Bogotá. Territorios, (38), 137-165. Doi: http://dx.doi.org/10.12804/revistas.urosario. edu.co/territorios/a.5909

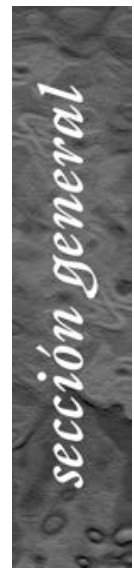

* PhD en Geografía de la Université Toulouse Le Mirail, Francia, 2012. Diplôme d'Etudes Approfondies - Master 2 en Sociología Université de Paris (Paris VIII), Francia, 1984. Master of Philosophy, Urban Design and Regional Planning Universtity of Edinburgh Escocia, Reino Unido, 1981. Socióloga de la Universidad Santo Tomás de Aquino, Bogotá, 1977. Pontificia Universidad Javeriana. Correo electrónico:ecorrale@javeriana.edu.co, ORCID: http://orcid.org/00000003-1981-2802.

* Bióloga de la Universidad de los Andes con énfasis en el área de ecología y organismos. Estudiante de tercer semestre del doctorado en Bioética. Magistra en Conservación y Uso $\Longrightarrow$ 
Palabras clave

Trade-off, sistema socio ecológico, servicios ecosistémicos, recurso hidrico.

\section{Keywords}

Trade-offs, Socio-ecological system, Ecosystem services, Water resource.

\section{Palavras-chave}

Trade-offs, Sistema Socioecológico, Serviços Ecossistêmicos, Recurso Hidrico.

\section{territarias 38}

\section{RESUMEN}

El sistema socio ecológico (en adelante, SSE) de la microcuenca de la quebrada San Cristóbal se encuentra localizado en el borde urbano rural de la localidad de Usaquén y la Reserva Forestal de los Cerros Orientales (Bogotá). Este SsE ha sido transformado por el fenómeno de crecimiento urbano, lo cual ha generado cambios en el uso del suelo y en la degradación ambiental del ecosistema, y ha afectado la biodiversidad y el servicio ecosistémico (en adelante, SE) de aprovisionamiento hídrico. Las decisiones o intervenciones humanas generan compromisos o tradeoff espaciales, temporales y sociales, que se analizan para el caso mencionado. La identificación y posterior análisis de estos trade-offs se hace a partir de diversas fuentes y consultas con diferentes actores. De esta manera se identifican los momentos clave que propiciaron la transformación del ecosistema, los efectos sobre el servicio ecosistémico a diferentes escalas espaciales y el balance de actores sociales que ganan o pierden en el proceso. Finalmente, a partir de las lecciones aprendidas, se encuentran variables y actores sociales clave, posibles escenarios y gestiones orientadas a la conservación o mejoramiento del SE.

\section{ABSTRACT}

San Cristobal's micro watershed is a socioecological system (SES), located in the rural-urban border of Usaquen and the Eastern Hills Forest Reserve (Bogota D.C). This SES has been transformed by the phenomenon of urban growth, generating changes in land use and environmental degradation of the ecosystem, affecting biodiversity and the ecosystem service (ES) of water supply. For the case mentioned, this study analyzes spatial, temporal and social trade-offs, which are created by human decisions or interventions. The identification and subsequent analysis of these trade-offs is made from various sources and consultations with different types of actors. Thus, key moments as what led to the transformation of the ecosystem, the effects on the ecosystem service at different spatial scales and the balance of social actors that won or lost in the process were identified. Based on the lessons learned, key variables and social actors, possible scenarios, and actions aimed at the conservation or improvement of the SE are identified.

\section{RESUMo}

O sistema socioecológico (SSE) da microbacia do riacho San Cristóbal, encontra-se localizado na beira urbana rural da localidade de Usaquén e a Reserva Florestal dos Cerros Orientais (Bogotá D.C.). Este SSE tem sido transformado pelo fenómeno de crescimento urbano, gerando mudanças no uso do solo e degradação ambiental do ecossistema, afetando a biodiversidade e o serviço ecossistêmico (SE) de aprovisionamento hídrico. As decisões ou intervenções humanas geram compromissos ou trade-offs, espaciais, temporais e sociais que se analisam para o caso mencionado. A identificação e posterior análise destes trade-offs se faz a partir de diversas fontes e consultas com diferentes tipos de atores. Assim se identificaram os momentos chave que propiciaram a transformação do ecossistema, os efeitos sobre o serviço ecossistêmico a diferentes escalas espaciais e o balanço de atores sociais que ganham ou perdem no processo. A partir das lições aprendidas, identificam-se variáveis e atores sociais chaves, possíveis cenários e ações orientadas à conservação ou melhoramento do SE. 


\section{Introducción}

Los servicios ecosistémicos (en adelante, $\mathrm{SE}$ ) son los beneficios que obtienen los humanos de los ecosistemas, los cuales soportan directa o indirectamente su supervivencia y calidad de vida (Díaz et al., 2011). En relación con la biodiversidad se “[...] reconoce su función como fuente de bienestar para la población, en la medida que de ella dependen los ciclos naturales que regulan el agua, el clima, la calidad del aire, la productividad del suelo que asegura la provisión de alimentos y muchos otros servicios ecosistémicos indispensables para la supervivencia humana" (Secretaría Distrital de Ambiente - SDA \& Conservación Internacional - CI, 2010, s. p.). Estos servicios son de gran relevancia para el bienestar humano, ya que a un ambiente sano le corresponden estándares altos de salud para las personas (Corredor, Fonseca \& Páez, 2012). Además, los SE se complementan entre sí y generan integridad socio ecológica, de manera que la afectación de un elemento podría llegar a influir en la totalidad del sistema (Reina Rozo, 2013).

Un importante servicio ecosistémico, característico de los ecosistemas de alta montaña, que son los encargados de la regulación hídrica, es el de aprovisionamiento de agua, definido como la capacidad que tiene el ecosistema de proveer un flujo hídrico continuo y de calidad, que puede ser usado para el consumo, el almacenamiento de agua y otros servicios importantes para el ser humano (Albán, 2007).
Ante una intervención humana, las relaciones entre los SE pueden beneficiar o perjudicar la existencia de otros: puede suceder que el mejoramiento en el mantenimiento de uno genere el mismo efecto sobre otro servicio, o que bien el énfasis en un servicio promueva la degradación de otro. Así, la consideración de los tradeoff - también llamados compromisos-, que resultan de los procesos de toma de decisiones en torno a la gestión de los SE, aporta elementos nuevos para el diseño de estrategias de manejo que permitan asegurar un flujo variado de los servicios y que satisfagan el bienestar humano (MartínLópez \& Montes, 2011).

Existen tres tipos de trade-off: los temporales, los espaciales y los interpersonales. Los trade-off temporales se refieren a los beneficios ahora y costos a largo plazo, y están fuertemente relacionados con el concepto de desarrollo sostenible, en el cual se debe tener precaución con las acciones generadas para satisfacer las necesidades del presente para no comprometer las necesidades de las generaciones del futuro. Los trade-offespaciales ocurren cuando hay un beneficio local y los costos de su uso se presentan en otro lugar, que puede ubicarse en el ámbito local, regional o global; estos son frecuentemente analizados en las cuencas hidrográficas, donde asegurar la conservación de los bosques y la calidad del agua de la cuenca alta del río permite el disfrute de una buena calidad del agua en la cuenca baja, lo cual genera un beneficio local a un costo determinado. De la misma de la Biodiversidad de la Pontificia Universidad Javeriana. Especialista en Docencia Universitaria de la Universidad El Bosque. Tiene experiencia en investigación en el área de medio ambiental con bio-indicadores de contaminación. Actualmente es investigadora y docente universitaria de la facultad de Ingeniería, en el programa de Ingeniería Ambiental. Correo electrónico: vivianaosorno@gmail.com,oRCID: http://orcid.org/00000002-0647-2151.

territarias 38

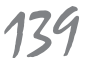


manera, si se contamina la cuenca alta por alguna actividad que beneficie a la población local, se afecta la calidad del agua en la cuenca baja y genera también un costo. Por último, los trade-off interpersonales o sociales hacen referencia un escenario donde unos actores ganan y otros pierden. Los actores sociales que se relacionan de diferentes maneras con los servicios que brinda el ecosistema y que tienen diferentes intereses en torno a estos pueden generar relaciones de conflicto y/o alianzas entre diversos actores sociales, lo que puede crear situaciones en las que unos actores ganan al aprovechar el SE en cuestión, mientras que otros pierden esa posibilidad (MartínLópez \& Montes, 2011).

El análisis de trade-off es una herramienta para conocer cómo funcionan las diferentes dinámicas sociales y ecológicas presentes en un socio-ecosistema, ya que proporcionan información sobre el balance entre servicios ecosistémicos y actores sociales vinculados con su uso y gestión. El incremento del uso de un servicio ecosistémico puede tener efectos como la disminución de otro y esto puede afectar de manera diferente el acceso de los actores a los SE. Para el caso de estudio, en donde el reto es conservar la biodiversidad y el recurso hídrico sin reducir el bienestar de las personas que habitan allí, estos trade-off se pueden dar entre el servicio ecosistémico de aprovisionamiento hídrico y los actores sociales identificados a diferentes escalas. Por eso el objetivo de esta investigación fue realizar un análisis de trade-off entre los distintos actores sociales, y entre ellos y el servicio ecosistémico de aprovisionamiento de calidad y cantidad de agua que presta la microcuenca de la quebrada San Cristóbal. Con esto se buscó aportar información de utilidad para la toma de decisiones que permitan la conservación de la biodiversidad urbano-rural de la zona.

\section{Descripción del sistema socio ecológico de la microcuenca de la quebrada San Cristóbal}

La microcuenca de la quebrada San Cristóbal se encuentra ubicada al norte de la localidad de Usaquén, que hace parte de la ciudad de Bogotá, la cual, además de ser la capital de Colombia, es la metrópoli con mayor aglomeración poblacional del país: 7571345 de personas, cifra que corresponde al $16 \%$ de la población nacional (Galvis, 2013). La microcuenca se encuentra dentro de la Unidad de Planeamiento Zonal (UPZ) número 11, que corresponde a San Cristóbal Norte (Secretaría Distrital de Planeación - SDP, 2009) (figura 1).

A escalas más amplias, o a escala regional, la microcuenca se encuentra en medio de los cerros orientales de Bogotá y hace parte de la subcuenca Torca-Guaymaral, que a su vez pertenece a l a cuenca del río Bogotá (Universidad El Bosque, 2013). A esta escala, la expansión urbana hacia los cerros, que involucra actividades de deforestación y cambios en el uso del suelo, genera conflictos por el uso y la tenencia de tierra (Bohórquez Alfonso, 2009). Todo esto afecta el servicio ecosistémico de aprovisionamiento de agua (Universidad

Elcy Corrales Roa, Viviana Osorno Acosta 
Figura 1. Ubicación de sSE de la microcuenca San Cristóbal
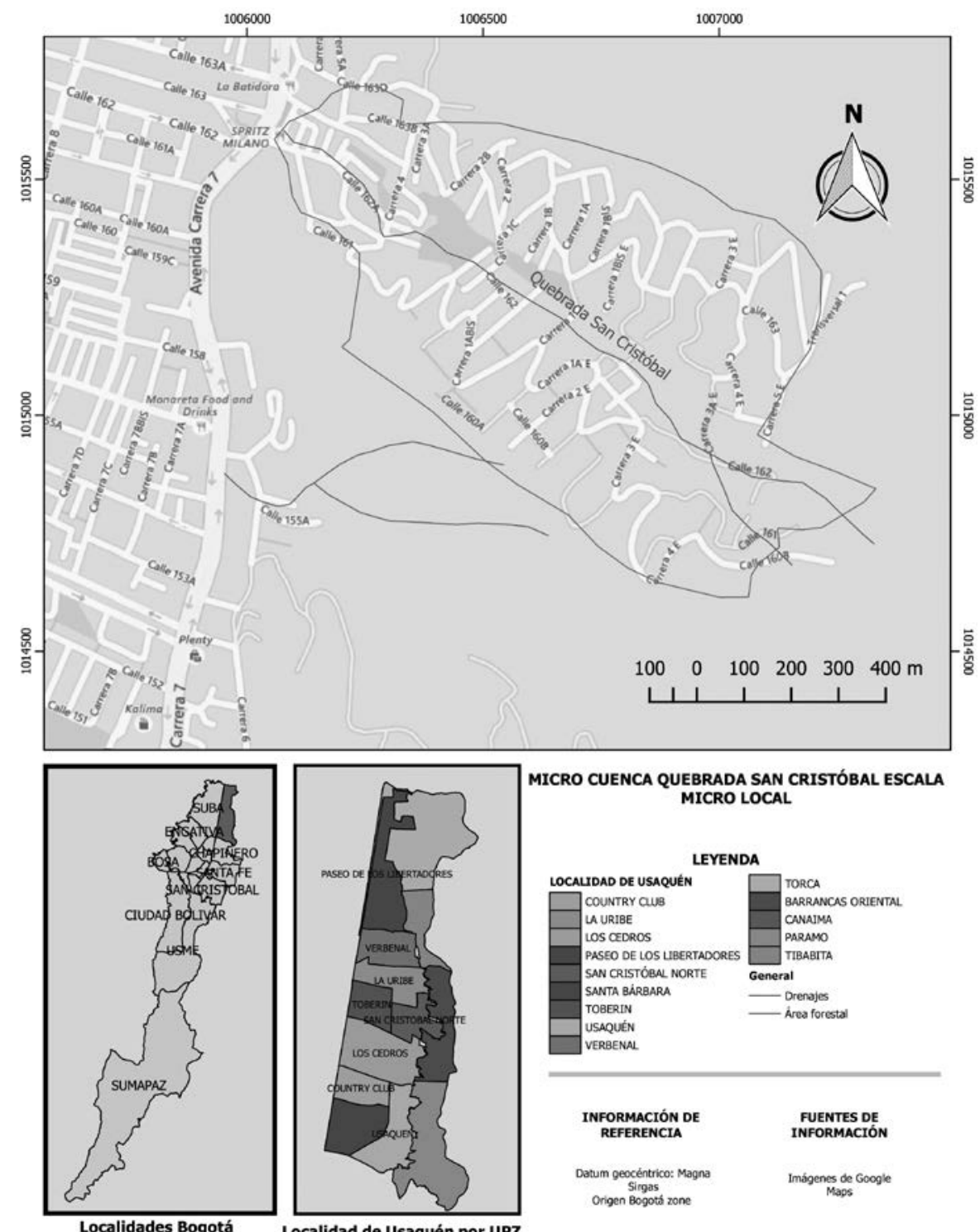
MICRO CUENCA QUEBRADA SAN CRISTÓBAL ESCALA
MICRO LOCAL

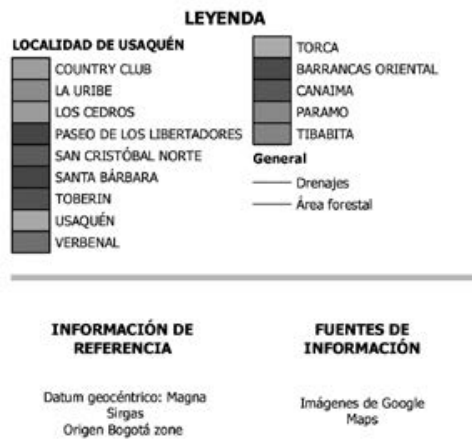

Fuente: Elaboración propia a partir de los datos recolectados en esta investigación. Para su composición se utilizó el programa libre y gratuito Qguis.

*A escala regional se presenta la ciudad de Bogotá; a escala local, el mapa de la localidad de Usaquén distribuido por UPZ; y a escala micro local, el mapa de la microcuenca de la quebrada San Cristóbal. 
El Bosque, 2014), es decir, el caudal se ve reducido debido a la intervención que se hace sobre el ciclo hidrológico, lo cual disminuye las corrientes hídricas que nutren la quebrada San Cristóbal (Tobón, 2009; Van Der Hammen, 1998).

La biodiversidad urbana, entendida como todas las especies que habitan e interactúan entre sí al interior de las ciudades (Secretaría Distrital de Ambiente y Conservación Internacional, 2010), es importante dentro y fuera del entorno urbano para una amplia variedad de bienes y servicios que son esenciales para la sostenibilidad económica, social y ambiental (Secretariat of the Convention on Biological Diversity, 2012). En el caso que aquí se trata, la pérdida de hábitat afecta la alta biodiversidad de fauna y flora que se encuentra en los cerros (Garzón Díaz, 2014), razón por la que se trazó un límite entre lo urbano y lo rural a través de la declaración de los cerros orientales como Reserva Forestal Protectora en el año 1977 (Secretaría Distrital de Planeación, 2007). Además, el mantenimiento de la biodiversidad de las zonas urbanas y de los servicios ecosistémicos asociados a ella depende en gran medida de toda el área de influencia, que es aquella escogida como corredor de conservación, entendiendo que esta "[...] zona es estratégica para el mantenimiento de la estructura y la función de los ecosistemas que sustentan la biodiversidad del Distrito Capital" (Secretaría Distrital de Ambiente y Conservación Internacional, 2010, s. p.).

A escala local también se afecta la sub-

territarias 38 142 aguas contaminadas con vertimientos de aguas residuales domésticas y residuos sólidos que vienen desde la parte alta y media de la quebrada San Cristóbal, y que se acumulan en la parte baja (Universidad El Bosque, 2013). Esto genera colmatación por sedimentación, aumenta los riesgos por inundación (Van Der Hammen, 1998) y causa pérdida de biodiversidad asociada a la subcuenca, incluyendo el humedal Torca-Guaymaral (Secretaría Distrital de Ambiente, 2009). En síntesis: todo esto afecta la calidad de vida de las personas que viven en la localidad de Usaquén.

A escala microlocal, algunos de los pobladores de la microcuenca se encuentran ubicados en viviendas construidas en la ronda de la quebrada o en el área declarada como "reserva forestal", lo que los categoriza como ilegales por invadir los espacios considerados públicos, según el Decreto 1504 de 1998 (Alcaldía Mayor de Bogotá, 1998). La cantidad de agua de la quebrada se mantiene principalmente por la llegada de aguas lluvias y por los vertimientos líquidos por parte de la población que habita en su ronda. Además, los pobladores que viven cerca a la quebrada pierden calidad de vida al enfrentarse a: riesgos en su salud, causados por plagas, enfermedades y malos olores; pérdida de sus viviendas, por deslizamientos, inundaciones, o reubicación por ilegalidad en sus predios; influencia en su felicidad, que está asociada a la belleza del entorno.

Las condiciones actuales del SSE de la microcuenca de la quebrada San Cristóbal son el reflejo de los procesos sociales 
mencionados, del inadecuado control e intervención por parte de las instituciones públicas, encargadas de hacer cumplir las normas, especialmente las establecidas para el manejo del recurso hídrico y la expansión urbana hacia los cerros orientales de Bogotá (Hospital de Usaquén, 2012). A partir de lo anterior surge la pregunta: ¿es posible conservar la biodiversidad del socio-ecosistema de la microcuenca San Cristóbal y su a vez mantener el servicio ecosistémico de aprovisionamiento hídrico sin disminuir el bienestar de sus habitantes?

\section{Metodología}

Para definir el SSE de la microcuenca de la quebrada San Cristóbal, se hizo una adaptación del método PARDI, denominado así por las iniciales que indican los pasos a seguir para la construcción participativa los modelos del sSE: Problema, Actores, Recursos, Dinámicas, Interacciones (Mathevet, Etienne \& Lynam, 2011). El propósito de esta metodología es modelar de forma participativa las dinámicas socio-ecológicas (Etienne, Du Toit \& Pollard, 2011). "PARDI sirve para construir colectivamente con los actores, una representación común de cómo funciona el territorio, mirado como un sistema socio-ecológico, según una problemática de desarrollo específica" (Fallot, 2013, p. 9).

Para la identificación y análisis de trade-off existentes en el SSE de la microcuenca de la quebrada San Cristóbal, además de la revisión de fuentes secundarias, se emplearon diversas herramientas de tipo cualitativo que se trabajaron con diferentes grupos de actores: (1) discusión con la Comisión Ambiental Local (CAL), (2) entrevistas a profundidad con informantes clave y (3) taller participativo con habitantes de la microcuenca de la quebrada San Cristóbal.

El grupo focal con el cual se llevó a cabo la discusión fue el de la CAL de Usaquén, conformado por representantes del sector público y de las comunidades (habitantes de los barrios de la localidad). Las comisiones son las instancias de coordinación que articulan las operaciones de los actores estratégicos de la localidad hacia el fortalecimiento de la gestión ambiental local bajo el principio de sostenibilidad; con esto buscan el mejoramiento de las condiciones ambientales y, por lo tanto, el de la calidad de vida de los habitantes. Algunos de los asistentes representan instituciones como la Alcaldía Local, la Junta Administradora Local, las secretarías distritales (Ambiente, Planeación, Hábitat, Salud), el Jardín Botánico, la Empresa de Acueducto y Alcantarillado, la Empresa de Aseo, universidades, colegios, juntas de acción comunal y representantes de organizaciones ambientales ante la Secretaría Distrital de Ambiente (Alcaldía Mayor de Bogotá, 2011). La discusión se llevó a cabo a partir de la metodología de Martín, López y Montes (2012), para apreciar de manera general las prioridades que los actores sociales perciben y establecen sobre el servicio ecosistémico de aprovisionamiento hídrico y sus componentes de calidad y cantidad. territarias 38

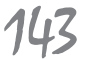


Se realizaron entrevistas a profundidad con cuatro informantes clave teniendo en cuenta dos criterios fundamentales: su saber experto sobre el recurso hídrico (calidad y cantidad) y su saber local en relación con la quebrada, bien por ser habitantes del sector o bien por manifestar interés sobre la situación ambiental de la microcuenca de la quebrada San Cristóbal Norte a través de trabajos de investigación previos. Los temas generales en estas entrevistas fueron: los cambios en la cantidad y calidad de agua en el tiempo (trade-off temporales), los cambios que ha tenido la calidad y cantidad de agua desde su nacimiento hasta su desembocadura (trade-off espaciales), y la identificación de actores y acciones en torno al agua (trade-off sociales). Estos temas permitieron determinar categorías de análisis para la información generada en las entrevistas, las cuales se organizaron con el software ATLAS.ti®.

Para complementar la información recolectada y visualizar la escala espacial a la que están funcionando los factores identificados en la entrevista, se usaron tres mapas que representan diferentes escalas a partir de las cuales se visualiza la microcuenca de la quebrada San Cristóbal. El primero muestra la microcuenca de la quebrada San Cristóbal, el segundo la misma microcuenca en medio de la zona urbana y los cerros orientales de Bogotá $\mathrm{y}$, por último, el tercero muestra un mapa por escalas espaciales (regional, local y microlocal). En estos mapas los actores entrevistados mostraron las variaciones en la calidad del agua desde su nacimiento hasta su desembocadura, la ubicación de algunos de sus usos, la distribución de los usuarios por estratos socioeconómicos y su percepción sobre la posición que ocupan (escala regional, local o microlocal) otros actores sociales en relación con el manejo y la intervención que estos hacen sobre el recurso hídrico de la quebrada San Cristóbal.

El taller con los habitantes de la microcuenca se realizó en el club de los abuelos "Los Conquistadores" en el barrio Cerro Norte (Usaquén), y tuvo como objetivo establecer, según la percepción de los asistentes, los posibles trade-off que pueden presentarse en el sistema socio ecológico de la microcuenca de la quebrada San Cristóbal, pero sin hacer explícito el concepto de trade-off. A este taller asistieron alrededor de 33 personas, en su mayoría adultos mayores del club y líderes comunitarios. Los grupos trabajaron simultáneamente en torno a los siguientes temas - todos relacionados con el recurso hídrico en la microcuenca-: la identificación de los SE y su problemática, la ubicación de usos y actividades en el mapa de la microcuenca San Cristóbal, y el análisis de escenarios futuros con base en el documento Participatory modelling of wellbeing tradeoffs in Coastal Kenya (P-Mowtick), en el que se busca encontrar una visión compartida y consensuada del futuro que los actores sociales desean para el socio ecosistema, a partir de talleres, análisis de escenarios y entrevistas (ESPA, 2012).

\section{territarias 38} 144 


\section{Resultados}

Los problemas relacionados con el SE de aprovisionamiento hídrico de la quebrada San Cristóbal identificados por la CAL fueron: (1) el incumplimiento de la normatividad por depositar aguas residuales sin tratar y construir en la ronda; (2) la falta de un acueducto alternativo o alcantarillado perimetral bajo conocimiento, control y vigilancia del Estado, que no se instala dadas las condiciones de ilegalidad de sus habitantes; (3) la poca articulación institucional; (4) la contaminación del agua por la mala disposición de residuos sólidos y por los vertimientos de aguas residuales a través de conexiones erradas; (5) la invasión de zonas; (6) el bajo conocimiento del mapa social y de contaminación; (7) la falta de mantenimiento de los centros de acopio de basuras, de la vegetación que rodea la quebrada, del sistema de acueducto, entre otros.

Una vez diagnosticado el problema se discute sobre las posibles soluciones, las cuales se enfocaron en la conservación, la protección del patrimonio ambiental y las líneas a seguir en torno a las construcciones y la articulación institucional. En cuanto a la construcción de infraestructura dentro del territorio hay ciertas necesidades, como la de servicios públicos (conducción de aguas negras), y otras que hay que evitar, como el incremento en la construcción de viviendas. Sin embargo, la infraestructura para atender la demanda de servicios públicos, como acueducto y alcantarillado, tiene altos costos de implementación y de mantenimiento que las instituciones públicas no están dispuestas a asumir, a pesar de que la inversión en este tipo de proyectos grandes para la población que vive en ronda de la quebrada solucionaría el problema de vertimientos de aguas residuales. Al referirse a la articulación institucional, se dice que la idea es hacer que converjan acciones e intervenir integralmente para que cada entidad coloque lo necesario desde su competencia. Se trata de una crisis de las entidades relacionada con la descentralización de las funciones, que resulta en una lectura e intervención diferentes sobre un mismo territorio y sobre una misma problemática planteada.

A lo anterior se proponen diferentes soluciones: (1) articular las instituciones buscando puntos de convergencia para solventar conflictos; (2) implementar la política en torno a la calidad de vida y al ambiente; (3) tener más voluntad política sobre la ejecución de la gestión pública; (4) el Estado debe contribuir a fortalecer el territorio desde lo colectivo y lo ambiental; (5) la academia es muy importante en estos ejercicios, pues tiene estudios que pueden brindar las herramientas para poder hacer propuestas clave en cada uno de los sectores.

Con el resultado de las entrevistas y al complementar la información recogida a través de las diferentes herramientas utilizadas para la identificación y posterior análisis de trade-off, se sistematizó y organizó la información por tipo de trade-off de la siguiente manera (tabla 1 ). territarias 38

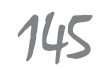


Tabla 1. Categorías según el tipo de trade-off

\begin{tabular}{|l|l|}
\hline \multirow{4}{*}{ Tradegorías } & Subcategorías \\
\hline \multirow{5}{*}{ Trade-off sociales } & Cambio en la calidad del agua \\
\cline { 2 - 3 } & Cambio en la cantidad del agua \\
\cline { 2 - 3 } & Futuro de la calidad y cantidad del agua \\
\hline \multirow{5}{*}{ Trade-off espaciales } & SE y los usuarios (uso del agua) \\
\cline { 2 - 3 } & Entre usuarios (acceso al agua) \\
\cline { 2 - 3 } & Instituciones y usuarios (normas y leyes sobre el recurso hídrico) \\
\hline & Relación quebrada - cerros orientales \\
\cline { 2 - 3 } & Relación quebrada - cobertura vegetal \\
\hline
\end{tabular}

Fuente: Elaboración propia.

El software ATLAS.ti ${ }^{\circledR}$ permitió generar dos diagramas por cada tipo de tradeoff: uno para el saber experto y otro para el saber local, con el fin de establecer similitudes y diferencias en las cuales se facilita la visualización de las respuestas por tipo de trade-off, como se muestra en el diagrama ejemplo para los trade-off espaciales (figuras 2 y 3 ).

Para los trade-off espaciales se establecieron similitudes y diferencias entre el saber local y el experto, las cuales sostienen la importancia que tiene la vegetación en el mantenimiento de la cantidad del agua que nace en las montañas de los cerros orientales de Bogotá: en cuanto a la calidad, determinan que la quebrada se empieza a contaminar donde inician los asentamientos humanos, y empeora cerca de su desembocadura. En general tienen la misma percepción y solo se diferencian en el nivel de detalle con el que explican los fenómenos ocurridos.

Al referirse a los cambios en la calidad y cantidad de agua, o trade-off temporales, el saber local explica las variaciones ocurridas desde la llegada de los primeros pobladores (añol975), mientras que los expertos solo tienen conocimiento desde el año 2012; sin embargo, confirman el deterioro continuo del SE de aprovisionamiento hídrico, sobre el que dicen que se puede recuperar en calidad y paisaje, pero no en cantidad en condiciones naturales.

Los trade-off sociales sí presentan grandes diferencias, especialmente en la percepción que tienen sobre las entidades. El saber local describe a las instituciones

Elcy Corrales Roa, Viviana Osorno Acosta 


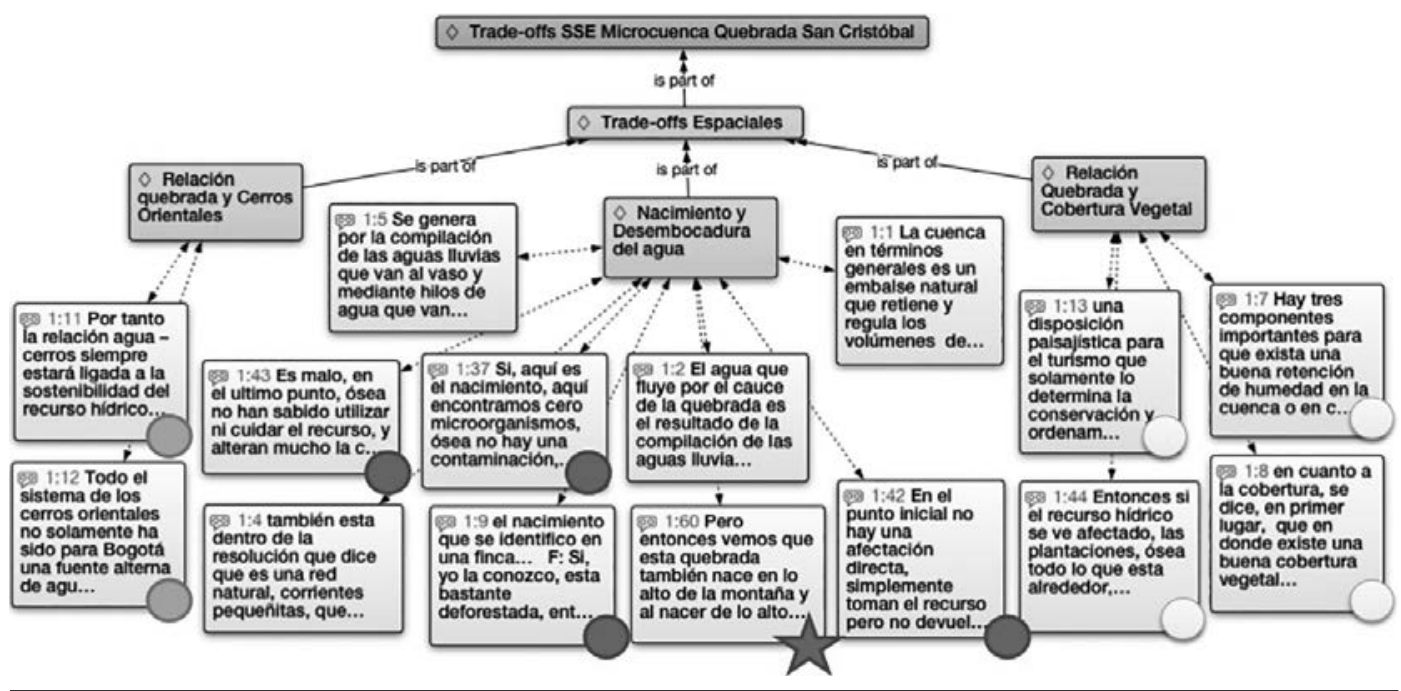

* Las cajas de color representan las categorías por tipo de trade-off, y las grises son fragmentos de la información recogida en las entrevistas. Los círculos representan los expertos en calidad y cantidad de agua, mientras que la estrella representa a la líder ambiental del Hospital de Usaquén. Los colores resaltan ideas similares entre los entrevistados.

públicas encargadas del recurso hídrico como ausentes en el territorio, mientras que los expertos se refieren a ellas como las encargadas de contribuir con gran parte de la solución. Todos mencionan la educación como un factor importante en el manejo del recurso, en el cual el saber local es más específico al diferenciar los habitantes según el interés y cuidado que puedan tener con la quebrada.

En cuanto a los resultados del taller con los habitantes locales, se identificaron como beneficios del ambiente o servicios ecosistémicos de la microcuenca de la quebrada San Cristóbal el buen vivir y los beneficios asociados al agua, como su uso para comer, lavar la ropa, regar las plantas

ANÁLISIS DE TRADE-OFF EN EL BORDE DE UNA RESERVA FORESTAL EN EL GRADIENTE URBANO-RURAL DE BOGOTÁ y huertas, descargar los sanitarios de los baños y darles de beber a los animales. Además proporcionaron información para encontrar el balance de trade-off temporales y sociales, como se registra en la tabla 2. Estos se repartieron en cuatro aspectos ecológicos: agua, biodiversidad, uso del suelo y área protegida.

El resultado de cada uno de estos trade-off se puede resumir al decir que los cambios que han ocurrido en el SSE de la microcuenca de la quebrada San Cristóbal han traído daños y beneficios. Con respecto al agua de la quebrada, por ejemplo, esta era abundante y de buena calidad, pero el acceso a ella era difícil, en cuanto tenían que hacer caminatas y filas para

territarios 38 147 


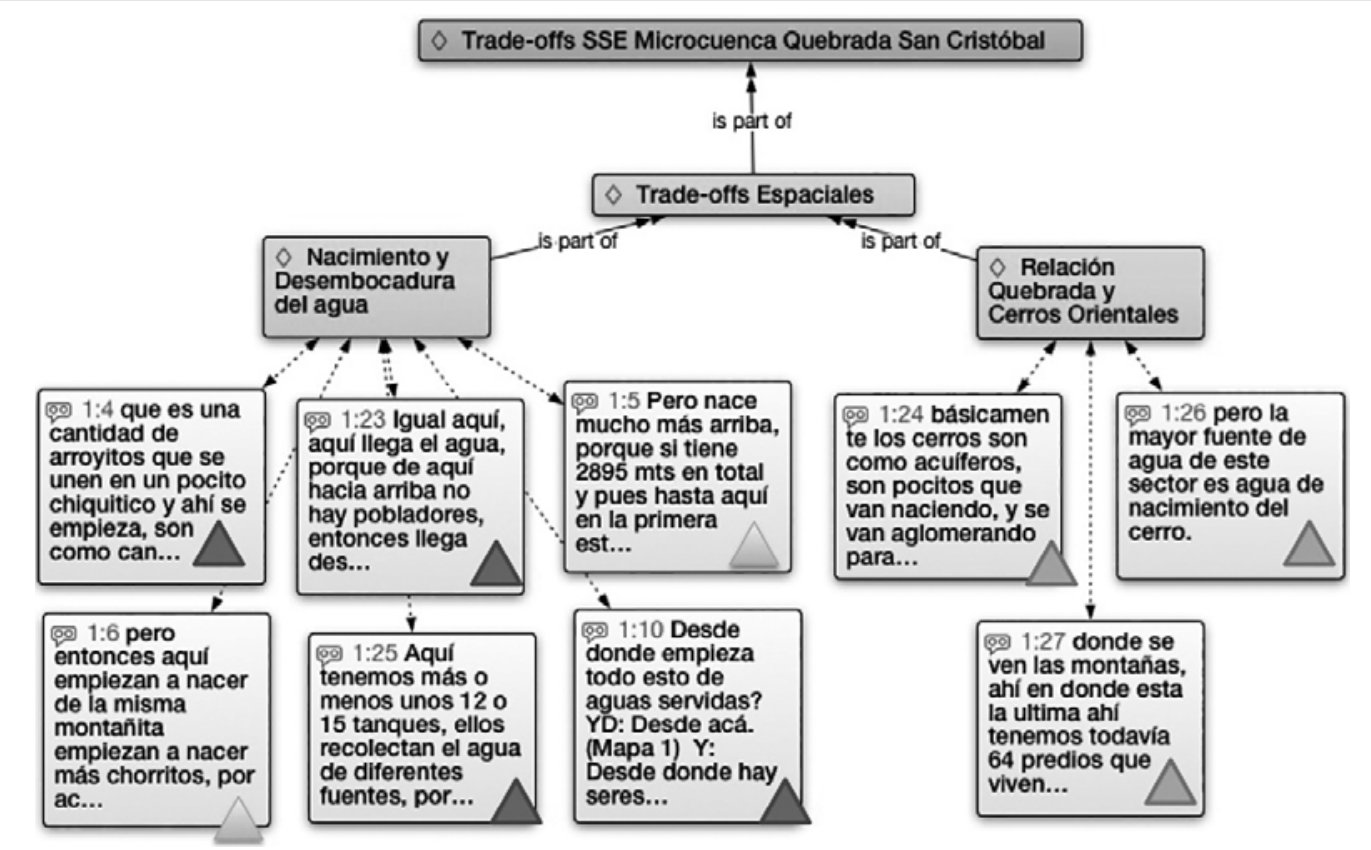

* Las cajas de color representan las categorías por tipo de trade-off, y las grises son fragmentos de la información recogida en las entrevistas. Los triángulos representan los líderes comunitarios entrevistados, y los colores resaltan ideas similares entre ellos.

llenar ollas y llevarlas a sus casas. Ahora las personas tienen agua en sus casas gracias al servicio de acueducto proveniente de tanques instalados en los años ochenta por la Empresa de Acueducto, Alcantarillado y Aseo de Bogotá (EAAB); pero al no tener alcantarillado, el uso de la quebrada cambió para ser un vertedero de aguas residuales. Por esta razón ya no es posible tomar agua directamente de la quebrada, pues produce enfermedades, a pesar de que algunos consideran esta agua natural e incluso mejor que la del acueducto que
Cuando los habitantes describen los cambios en la biodiversidad, hablan del antes como un lugar con gran variedad de plantas y animales, mientras que ahora dicen que hay pocos árboles. En el presente identifican a las mascotas como los animales más abundantes, considerando que afectan a todos los habitantes en tanto no hay árboles, ni animales como aves que alegren a las personas. En cuanto a los cambios en el uso del suelo, se hace evidente el proceso de transformación de rural a urbano, lo que consideran bueno por tener carreteras por donde transitar

Elcy Corrales Roa, Viviana Osorno Acosta 
Tabla 2. Identificación participativa de trade-off temporales y sociales

\begin{tabular}{|c|c|c|c|c|c|c|}
\hline $\begin{array}{c}\text { Característica } \\
\text { Aspecto } \\
\text { ecológico }\end{array}$ & Antes & Ahora & $\begin{array}{c}\text { Causas de } \\
\text { cambio }\end{array}$ & $\begin{array}{l}\text { Actores } \\
\text { sociales }\end{array}$ & Beneficia a: $(+)$ & Hace daño a: (-) \\
\hline $\begin{array}{l}\text { Agua (calidad } \\
\text { y cantidad) }\end{array}$ & $\begin{array}{l}\text { Era clara y limpia. Sufrían } \\
\text { antes por el agua. Hacían } \\
\text { turnos para los lavaderos. Se } \\
\text { iba a la represa y a los pozos } \\
\text { para llenar las ollas. Con esa } \\
\text { agua se cocinaba. }\end{array}$ & $\begin{array}{l}\text { Viene sucia; "viene } \\
\text { amarilla, pero de } \\
\text { todos modos yo la } \\
\text { hiervo". Ahora viene } \\
\text { del acueducto que fue } \\
\text { instalado hace } 25 \text { o } 30 \\
\text { años. }\end{array}$ & $\begin{array}{l}\text { Debido al } \\
\text { acueducto } \\
\text { se acabaron } \\
\text { los pozos de } \\
\text { agua por las } \\
\text { construcciones } \\
\text { y la canalización } \\
\text { en } 1983 \text {. }\end{array}$ & $\begin{array}{l}\text { Acueducto, } \\
\text { constructores } \\
\text { de viviendas. }\end{array}$ & $\begin{array}{l}\text { Mucha gente } \\
\text { que tiene agua } \\
\text { en sus casas. } \\
\text { No hay que } \\
\text { acarrearla. Se } \\
\text { tiene agua para } \\
\text { comer (cocinar) } \\
\text { y bañarse. }\end{array}$ & $\begin{array}{l}\text { Quienes beben } \\
\text { el agua de la } \\
\text { quebrada que } \\
\text { tiene amebas } \\
\text { y lombrices } \\
\text { (produce } \\
\text { amebiasis). } \\
\text { El agua de la } \\
\text { naturaleza es } \\
\text { mejor que la } \\
\text { del acueducto, } \\
\text { la mejor es la } \\
\text { del nacimiento } \\
\text { porque no tiene } \\
\text { químicos. }\end{array}$ \\
\hline
\end{tabular}




\begin{tabular}{|c|c|c|c|c|c|c|}
\hline $\begin{array}{c}\text { Característica } \\
\text { Aspecto } \\
\text { ecológico }\end{array}$ & Antes & Ahora & $\begin{array}{c}\text { Causas de } \\
\text { cambio }\end{array}$ & $\begin{array}{l}\text { Actores } \\
\text { sociales }\end{array}$ & Beneficia a: (+) & Hace daño a: (-) \\
\hline $\begin{array}{l}\text { Área } \\
\text { protegida } \\
\text { (bosque de } \\
\text { los cerros } \\
\text { orientales) }\end{array}$ & $\begin{array}{l}\text { Antes había mucho árbol } \\
\text { nativo... también se } \\
\text { sembraba. }\end{array}$ & $\begin{array}{l}\text { Ya no hay tanto árbol: } \\
\text { hay algunos arriba, } \\
\text { pero no al borde de la } \\
\text { quebrada. }\end{array}$ & $\begin{array}{l}\text { Las } \\
\text { construcciones } \\
\text { desde hace } \\
\text { como } 65 \text { años. } \\
\text { Antes había } \\
\text { gente que se } \\
\text { "robaba los } \\
\text { árboles porque } \\
\text { necesitaba la } \\
\text { madera" para } \\
\text { venderla porque } \\
\text { no trabajaba. }\end{array}$ & $\begin{array}{l}\text { Los que } \\
\text { cortan } \\
\text { árboles para } \\
\text { madera, para } \\
\text { hacer casas } \\
\text { o para pagar } \\
\text { el vicio, y } \\
\text { constructores. }\end{array}$ & $\begin{array}{l}\text { Los árboles son } \\
\text { los que hacen } \\
\text { que haya más } \\
\text { cantidad de } \\
\text { agua. }\end{array}$ & $\begin{array}{l}\text { A todos porque } \\
\text { los bosques dan } \\
\text { el agua y si no } \\
\text { hay se "favorece } \\
\text { la sequedad de la } \\
\text { tierra". Hace falta } \\
\text { para purificar el } \\
\text { aire, la atmósfera. }\end{array}$ \\
\hline
\end{tabular}

Fuente: Elaboración propia.

\section{territarias 38}

en vehículos, pero malo al no tener suelo donde sembrar. Además le atribuyen la inseguridad actual al aumento en la llegada de nuevas personas provenientes de diferentes ciudades del país.

El último aspecto es el área protegida, respecto al cual recuerdan que antes había más árboles, pero, por las construcciones y la necesidad de conseguir madera, estos han disminuido cada vez más. Esta situación afecta a todos los habitantes, ya que los árboles limpian el aire y ayudan a mantener una buena cantidad de agua.

Con relación a los trade-off espaciales, identificados y organizados por colores en el taller participativo, los resultados se presentan en la figura 4 , donde se muestra la ubicación de las actividades actuales a lo largo de la microcuenca de la quebrada San Cristóbal, a través de fotografías. En todos los casos se trata de extracción de agua diferente de la que es conducida por el acueducto. En la parte alta de la quebrada se hace extracción de agua para preparar alimentos, para regar jardines o cultivos y darle de beber al ganado; en la parte media de la quebrada se usa para lavar ropa y carros; la deposición de aguas residuales empieza desde la parte media y se vuelve más frecuente en la parte baja, donde hay más concentración de viviendas; y el depósito de residuos sólidos ocurre con mayor frecuencia en la parte baja de la quebrada. Hay una clara diferenciación en la extracción, los usos y las consecuencias sobre el SE en los distintos niveles o espacios de la cuenca.

En la figura 5 se representa el uso y la modificación del recurso hídrico por parte de los grupos de actores sociales identificados a través del proceso de investigación, dentro de los cuales se destacan los siguientes: instituciones no gubernamentales, empresas privadas, instituciones públicas (Alcaldía Local, Junta Administradora Local, secretarías distritales, Jardín Botánico, 
Figura 4. Ubicación de las actividades y usos actuales dentro de la microcuenca de la quebrada San Cristóbal

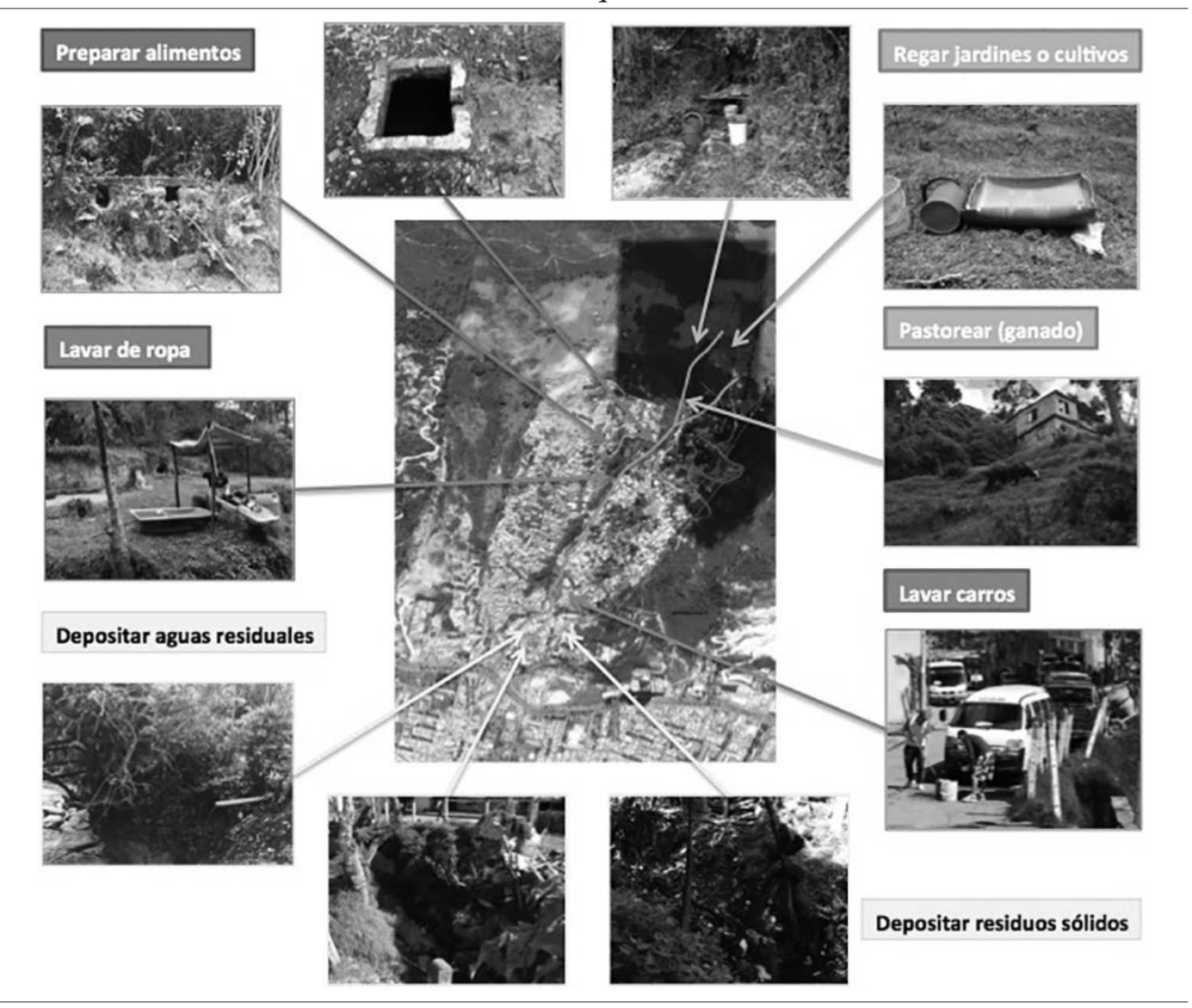

Fuente: Fotografías capturadas en la quebrada San Cristóbal.

* Se clasifican por colores sobre fotografías tomadas en la zona de estudio.

Empresa de Acueducto y Alcantarillado), instituciones educativas y organizaciones comunales (Fundación Manigua, grupo los Conquistadores) y los usuarios clasificados principalmente en ganaderos, agricultores y residentes urbanos.
Dentro de los residentes urbanos se presentan diferencias según el grado de apropiación que tienen sobre el territorio y su valoración sobre el SE y sus atributos: los primeros pobladores son los más interesados en conservar el SE de aprovisionamiento hídrico, así como otros SE 
Figura 5. Representación gráfica del uso y la modificación del recurso a través de las diferentes acciones y procesos que ejercen algunos grupos de actores sociales en el sSE de la microcuenca San Cristóbal

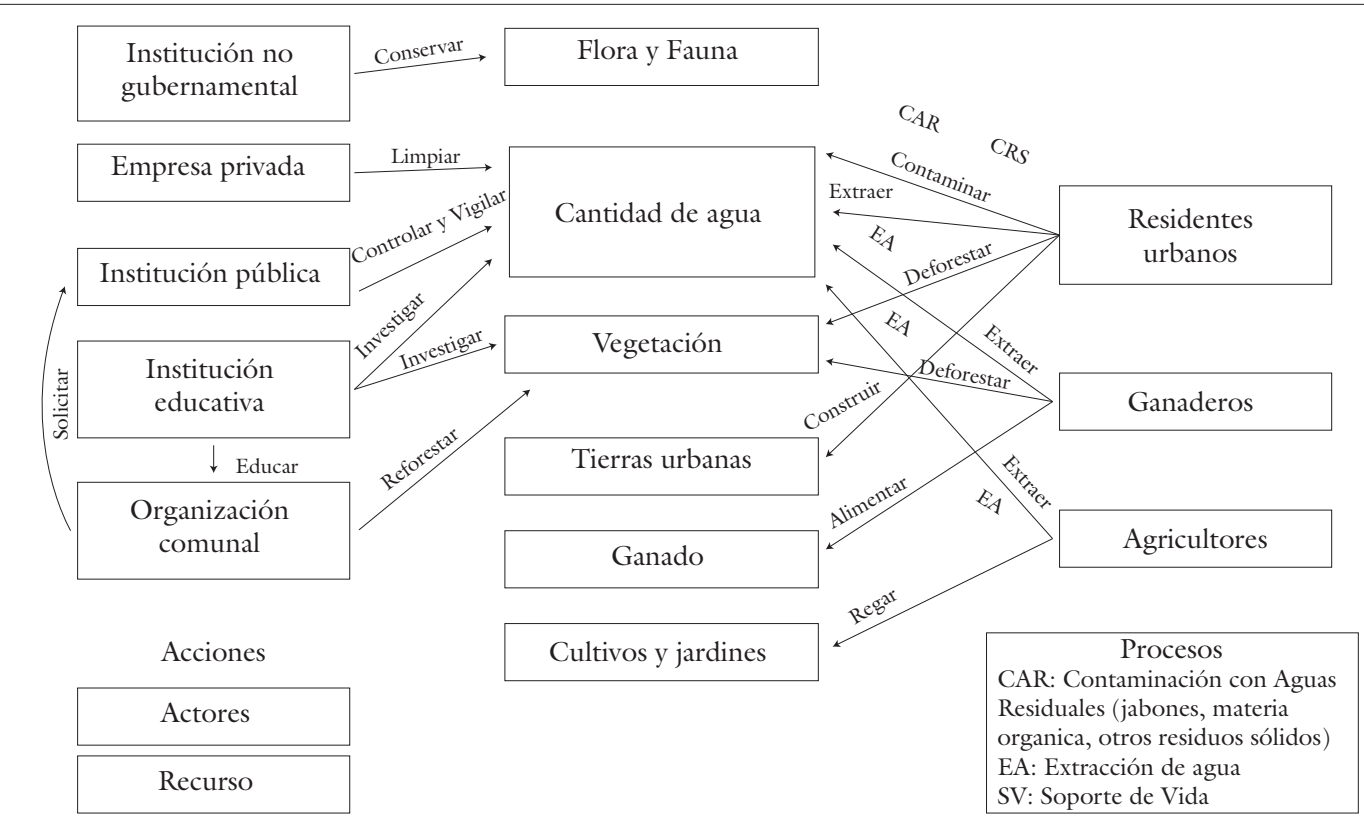

Fuente: *Adaptado del método PARDI.

asociados. Esto no ocurre con las nuevas generaciones o con los llamados pobladores flotantes, es decir, las personas que viven en arriendo dentro de alguno de los niveles de las casas dedicados a esta actividad arrendataria. Otra diferencia está en su ubicación con respecto a la quebrada: unos son los habitantes que viven en ronda y que tienen servicio de acueducto pero no de alcantarillado, y otros, los que viven en los barrios legalizados, un poco más lejos de la quebrada y que cuentan con todos los servicios públicos. Además, estos usuarios pueden estar distribuidos en la parte alta, media y baja de la quebrada San Cristóbal.

En relación con el ejercicio de discusión sobre escenarios construidos previamente, a partir del proceso que se ha vivido en la zona y de los datos de las entrevistas con los diversos actores se desarrolló una discusión que giró en torno a: cambios en el SE, ganadores y perdedores. Los resultados se consignan en la tabla 3 . En ella los participantes describen que, si las dinámicas actuales continúan y dejan un futuro como el propuesto en el escenario 2, 
Tabla 3. Posibles escenarios

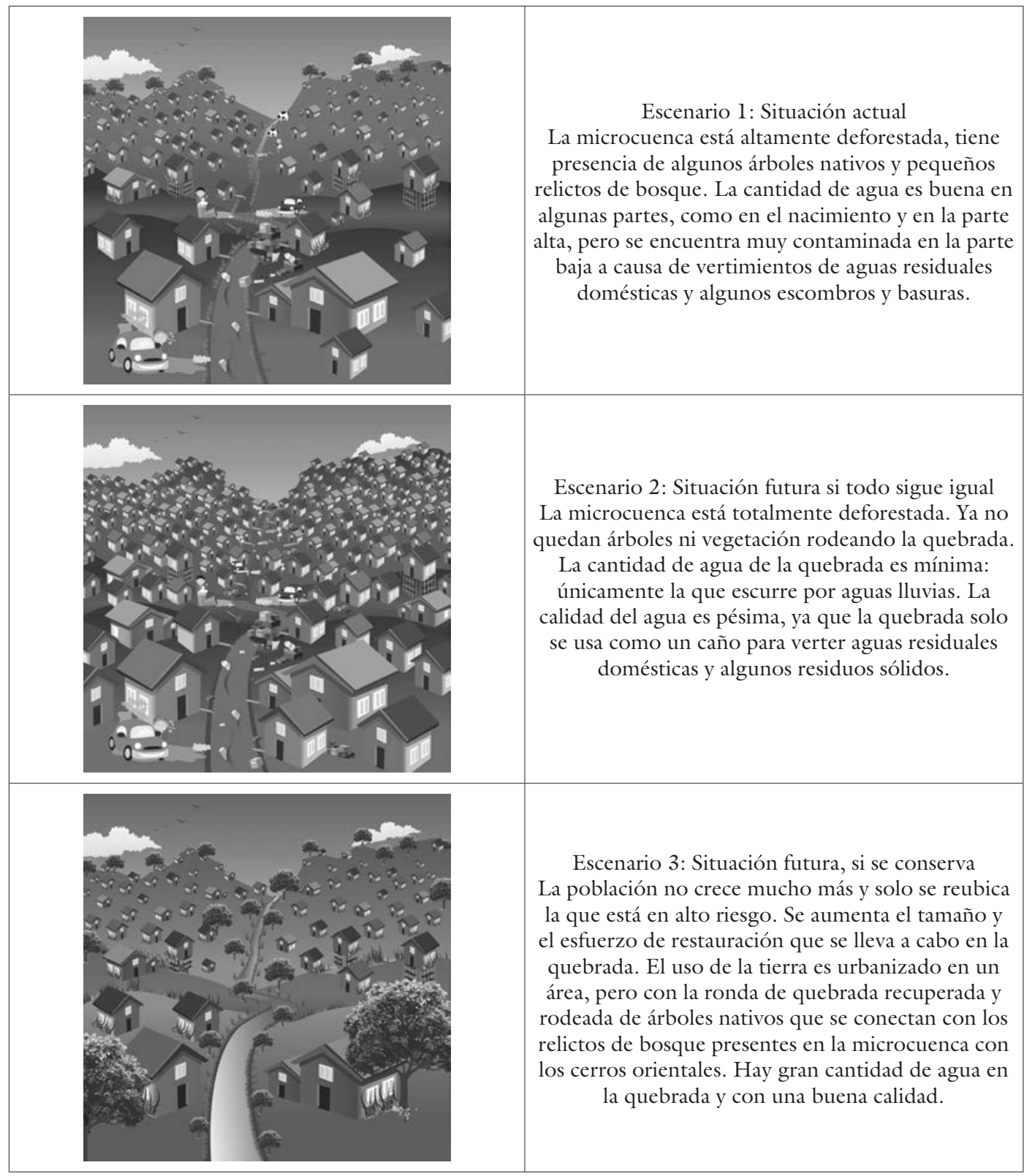

Fuente: Elaboración propia. 
la posibilidad de prolongar los usos actuales de la quebrada se hace cada vez menor, así como los SE van disminuyendo. En contraste, aquellos participantes tienen claro que si la situación actual mejorara, como en el escenario 3 , algunos usos de la quebrada se podrían restablecer (preparar alimentos, lavar, regar), así como también sería necesaria la eliminación de otros (depositar aguas residuales y residuos sólidos), y con esto se asegura que muchos SE puedan incrementar su futuro de conservación.

Las actividades y el aprovechamiento de los servicios ecosistémicos se calificaron en cada uno de los escenarios como se muestra en la tabla 4.

\section{Análisis de trade-off en el sistema socio ecológico de la microcuenca de la quebrada San Cristóbal}

\subsection{Trade-off temporales}

Los cambios en la cantidad de agua están fuertemente relacionados con lo que ha sucedido a escala regional, como la transformación de los cerros orientales como consecuencia de la expansión urbana. Las causas son de diverso tipo, como el desplazamiento de campesinos a la ciudad, el desmonte de los cerros por minería, el déficit de vivienda en la ciudad, el precio del suelo, el incumplimiento de la normatividad por parte de diversos actores (habitantes, vendedores ilegales de terreno, recicladores, ganaderos, pequeños agricultores, entre

\section{territarias 38} 154 lancia por parte del Estado para que estas normas se cumplan. Lo anterior da como resultado la deforestación de los cerros y con esto la disminución de la oferta hídrica.

Lo mismo sucedió a escala microlocal, ya que hasta el año 1950 la microcuenca tenía vegetación original: estaba compuesta por chuscales, romerón, encenillos, piñas, aguadijas, musgos y líquenes, entre otros. Para la movilidad de la pequeña población de ese entonces, solo se contaba con dos senderos que recorrían desde la parte alta de la cuenca hasta la carrera Séptima. Lo que permite suponer que la calidad del agua para estos años mantenía sus condiciones por la ausencia casi total de población. Además, es posible que la presencia de esa cobertura vegetal contribuyera a regular el ciclo hidrológico de la microcuenca de la quebrada San Cristóbal, ayudando a mantener la disponibilidad de agua dentro y fuera de la microcuenca (Universidad El Bosque, 2014). Ahora, a pesar de que la microcuenca esta urbanizada, aún se encuentran parches de bosques nativos, rastrojos altos y bajos con especies colonizadoras, oportunistas, de rápido crecimiento; bejucos y gramíneas. En las zonas urbanas desarrolladas, en las zonas verdes y en los parques hay un predominio en el uso de especies ornamentales tanto nativas como exóticas (Hospital de Usaquén, E.S.E, 2012).

Esta pérdida de cobertura vegetal pudo ser controlada cuando se declaró a los cerros como Reserva Forestal Protectora en el año 1977 (SDA, 2007). Pero los cambios en la normativa para la delimi-

Elcy Corrales Roa, Viviana Osorno Acosta 
Tabla 4. Actividades y aprovechamiento de servicios ecosistémicos en diferentes escenarios

\begin{tabular}{|c|c|c|c|}
\hline \multirow{2}{*}{$\begin{array}{l}\text { Actividades relacionadas } \\
\text { con el uso del agua }\end{array}$} & \multicolumn{3}{|c|}{ Escenarios } \\
\hline & 1. Actual & 2. Futuro - Todo sigue igual & 3. Futuro de conservación \\
\hline Preparar alimentos & $\downarrow$ & $\downarrow \downarrow$ & $\uparrow \uparrow$ \\
\hline Lavar ropa y carros & $\downarrow$ & $\downarrow$ & $\uparrow \uparrow$ \\
\hline Pastorear (ganado) & $\leftrightarrow$ & $\downarrow \downarrow$ & $\downarrow$ \\
\hline Depositar aguas residuales & $\leftrightarrow$ & $\downarrow$ & $\downarrow$ \\
\hline Depositar residuos sólidos & $\leftrightarrow$ & $\downarrow$ & $\downarrow$ \\
\hline Regar jardines o cultivos & $\downarrow$ & $\downarrow$ & $\uparrow$ \\
\hline Construir viviendas & $\downarrow$ & $\uparrow \uparrow$ & $\downarrow \downarrow$ \\
\hline Agua para baños & $\downarrow$ & $\downarrow \downarrow$ & $\uparrow \uparrow$ \\
\hline \multicolumn{4}{|l|}{ SE (beneficios del ambiente) } \\
\hline Paisaje & $\leftrightarrow$ & $\downarrow$ & $\uparrow$ \\
\hline Aire limpio & $\leftrightarrow$ & $\downarrow \downarrow$ & $\uparrow \uparrow$ \\
\hline Suelo & $\leftrightarrow$ & $\downarrow$ & $\uparrow$ \\
\hline Medicamentos & $\leftrightarrow$ & $\downarrow$ & $\uparrow \uparrow$ \\
\hline Actividades & $\leftrightarrow$ & $\downarrow$ & $\uparrow \uparrow$ \\
\hline Alimentos & $\leftrightarrow$ & $\downarrow$ & $\uparrow \uparrow$ \\
\hline Tranquilidad & $\downarrow$ & $\downarrow$ & $\uparrow$ \\
\hline Salud & $\downarrow$ & $\downarrow$ & $\uparrow \uparrow$ \\
\hline Vivienda & $\leftrightarrow$ & $\downarrow$ & $\uparrow$ \\
\hline Seguridad & $\downarrow$ & $\downarrow \downarrow$ & $\uparrow \uparrow$ \\
\hline
\end{tabular}

Fuente:

*Los escenarios fueron categorizados de la siguiente manera: $\uparrow \uparrow:$ Aumenta mucho; $\uparrow:$ Aumenta poco; $\leftrightarrow:$ se mantiene constante; $\downarrow$ : disminuye poco; $\downarrow \downarrow$ : disminuye mucho.

tación y el ordenamiento de estos cerros han causado confusión y problemas para el control y manejo de la urbanización en el borde urbano-rural. En el año 1990,

ANÁLISIS DE TRADE-OFF EN EL BORDE DE UNA RESERVA FORESTAL EN EL GRADIENTE URBANO-RURAL DE BOGOTÁ los barrios de la microcuenca pasaron a ser suelo urbano, pero más adelante, en el año 2004 , volvieron a quedar en medio de la reserva forestal debido a la creación del

territarios 38

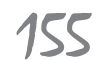


corredor ecológico de borde. En el año 2005, con la Resolución 463, la microcuenca nuevamente entró a ser parte del suelo clasificado como urbano, al crear una franja de adecuación que no se reglamentó en el 2006 y que hizo que para el año 2007 la microcuenca aún se encontrara en la zona de reserva (SDP, 2007). En el año 2015 se corrigieron estas imprecisiones cartográficas para establecer la delimitación según la declarada en 1977, es decir que ahora se encuentra en suelo urbano y en zona de reserva (Secretaría Distrital de Planeación - SDP, 2015).

La calidad del agua también se ve afectada en la medida en que la población aumenta alrededor de la quebrada y, con esta, sus diferentes usos, especialmente cuando se instala el servicio de acueducto en la mayoría de barrios ubicados en la microcuenca. A partir de ese momento la extracción de agua deja de ser para consumo directo y la quebrada se convierte en un vertedero de aguas residuales domésticas. Esto cambia la percepción de los habitantes, lo que perjudica aun más la quebrada, ya que se usa para botar residuos sólidos como basuras y escombros.

Los actores sociales directos, como líderes comunitarios y otros habitantes de la microcuenca, coinciden en que la llegada del acueducto y la facilidad de tener agua en sus casas conduce al descuido de la fuente de agua original. Sin embargo, manifiestan que quisieran recuperar la quebrada, devolverla a lo que era antes: un entorno agradable de encuentro y disfrute.

\section{territarias 38}

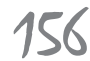

Los expertos en calidad y cantidad de agua confirman el cambio negativo que ha tenido la quebrada San Cristóbal, el cual no ha mejorado en los últimos años. Incluso tienen posiciones encontradas frente a la posibilidad de recuperación, al considerar que su caudal se mantiene por la llegada de aguas lluvias y los desagües domésticos que incrementan su nivel de contaminación. Explican que recuperar el suelo después de procesos de pavimentación es complicado, pero se puede recuperar la quebrada en términos de paisaje, calidad de agua y solucionar parte de la problemática ambiental. En todo caso, los expertos consideran que la participación de la comunidad es importante en el proceso de recuperación de la calidad y cantidad del agua de la quebrada, gestionando acciones como la solicitud a la Secretaría Distrital de Ambiente de la reforestación de la ronda de la quebrada y los canales que capten aguas negras.

Los trade-off temporales muestran cómo el servicio de aprovisionamiento hídrico, que benefició y permitió la llegada y el establecimiento de los primeros pobladores a la microcuenca, se fue deteriorando a causa de la contaminación, la deforestación y la captación descontrolada del agua, impidiendo así que se recupere bajo condiciones naturales. Los costos futuros se manifiestan en una quebrada cada día más contaminada y con menos agua que afecta la biodiversidad que aún sobrevive en la microcuenca y que disminuye la calidad de vida de sus habitantes.

A diferentes escalas sucede algo similar: a escala local, la subcuenca Torca-

Elcy Corrales Roa, Viviana Osorno Acosta 
Guaymaral continuará recibiendo menos agua y más carga de contaminantes. A escala regional, la microcuenca perderá las propiedades ecológicas que le permiten refugiar algunas especies que se encuentran en esos espacios de transición entre lo urbano y lo rural, que a su vez mantienen la conexión con los cerros orientales de Bogotá.

\subsection{Trade-off espaciales}

Los trade-off espaciales se dan a escalas amplias o regionales desde los cerros orientales, donde nacen las quebradas, hasta donde llegan sus aguas, como el río Bogotá; a escalas medias, como la localidad de Usaquén, que conecta la quebrada San Cristóbal con la subcuenca Toca-Guaymaral y el humedal del mismo nombre; y a escalas locales, entre las cuencas alta, media y baja.

Los cerros orientales son una fuente hídrica y contribuyen a la formación de arroyos que permiten la creación y el mantenimiento de la quebrada San Cristóbal. Sin embargo, los cambios en los usos del suelo - como la urbanización-involucran la deforestación de esta cobertura vegetal y causan una disminución en la retención de humedad, lo que da como resultado un menor caudal. Además, desde que hay presencia humana, hay afectaciones por el uso que las personas le dan a la quebrada, y esto hace que la calidad del agua disminuya casi desde su nacimiento y a lo largo de la quebrada, y que empeore en la parte media y, más aún, en la parte baja. Esto, además, afecta al río Torca y más adelante al humedal Torca-Guaymaral.

La vegetación en los cerros y en la microcuenca es importante para la generación y conservación del agua. Este resultado exige condiciones como que no se vea afectado el ciclo hidrológico y que no se altere la tasa de evapotranspiración producida por el cambio de uso del suelo. Esta última situación se puede presentar cuando el bosque es reemplazado por vegetación rastrera (por ejemplo, pastos), con una menor cantidad de hojas y de menor cobertura. Así se generan variaciones considerables en la infiltración y en las propiedades hidrofísicas del suelo, lo cual resulta en una menor recarga del agua del suelo y de los acuíferos en los periodos de lluvia, y deja los suelos rápidamente expuestos a condiciones de sequedad (Tobón, 2009). En general se reduce la captación y la disponibilidad de agua, lo que cambia el flujo de vapor y el drenaje superficial y subterráneo (Ataroff \& Rada, 2000).

Esta relación entre la vegetación y el agua es reconocida por los habitantes de la quebrada, quienes están bien al tanto de las especies de plantas nativas que había antes y hay ahora; entienden que cuando hay árboles, hay agua, y viceversa. Incuso las líderes comunitarias hablan de que el nacimiento de agua viene de los cerros orientales de Bogotá. Además, algunos habitantes de la microcuenca piden que se les regale plantas para reforestar la ronda o para sembrar cerca de sus casas, pues así pueden prestar otros servicios ecosistémicos, como aire puro y alegría al estar en territarias 38

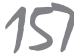


un espacio verde. Esto refuerza la idea de incrementar el sentido de apropiación del territorio por parte de los habitantes, tanto de la microcuenca, como de la ciudad de Bogotá, en tanto se entiendan los beneficios o servicios ecosistémicos que prestan los cerros orientales.

Las condiciones del SSE a diferentes escalas espaciales se resumen en que lo que pasa en la cuenca alta (beneficios acá) va a repercutir con mayor fuerza en las cuencas media y baja (costos allá); así como a escala microlocal los efectos son mayores que en las escalas local y regional. No pasa lo mismo con la calidad de vida de los habitantes o con las intervenciones institucionales. La primera está asociada al uso del recurso de forma que, probablemente, si los de la cuenca alta disminuyen un poco su calidad de vida, no afecten tanto a los de las cuencas media y baja; en cuanto a las intervenciones por parte de las instituciones públicas y privadas, se hacen mayores en la medida en que se van necesitando, siendo prioritarias las que ejercen un mayor efecto sobre las otras escalas, como es el caso de la cuenca alta y la escala microlocal. Es decir que si se soluciona parte de las problemáticas a estas escalas, se reflejará una mejora de los problemas a otras escalas.

\subsection{Trade-off sociales}

El proceso de identificación de actores sociales se profundiza cuando se descubren diferencias dentro de un mismo tipo de actor, que por lo general no son percibidas por los otros actores sociales. Es el caso de los habitantes de un área específica, como la microcuenca, que suelen llevar el nombre de comunidad por parte de las instituciones públicas e impiden que se reconozcan sus diferencias internas, ya que tienen percepciones e intereses distintos sobre el SSE. Lo mismo sucede con las instituciones: actúan y se involucran de diferentes maneras dentro del territorio.

Los trade-off sociales se pueden notar de diferentes maneras: entre SE y usuarios, entre SE y calidad de vida, entre usuarios, y entre usuarios e instituciones. Aquí es evidente cómo el uso que se le da a la quebrada y a la cobertura vegetal que la rodea afecta no solo la calidad y cantidad de agua, sino también los diferentes usuarios que hacen usos de esta.

Entre los SE y los usuarios se presentan múltiples trade-off que se relacionan directamente con las características del usuario. Los ganaderos extraen agua para darles de beber a los animales. Dicha extracción puede hacerse por medio de recipientes o llevando directamente el ganado a la quebrada; en el primer caso se disminuye la cantidad de agua de la quebrada por captación, mientras que en el segundo, además del consumo por parte del ganado, podría afectarse su calidad cuando los animales pisan o defecan dentro o cerca de la quebrada. Los agricultores generan cambios en el uso del suelo: deforestan la vegetación nativa para sembrar y también extraen agua para riego. Ambas actividades generan reducción en la cantidad de agua.

Los usuarios que habitan en la ronda de la quebrada y que no tienen servicio de

Elcy Corrales Roa, Viviana Osorno Acosta 
acueducto y alcantarillado extraen el agua desde su nacimiento para el consumo, para preparar alimentos, para descargar el baño, para lavar utensilios, entre otras actividades donde el agua se transforma, contamina y se depositada a la quebrada, lo cual baja la calidad del recurso. Los usuarios que sí tienen servicio de acueducto, pero no de alcantarillado, ejercen ese mismo proceso de transformación y vertimiento del agua a la quebrada por medio de tuberías y conexiones cerradas desde sus casas hacia la quebrada. De los usuarios mencionados, aquellos que viven en la parte alta son los que hacen la mayor extracción de agua limpia, mientras que los de las partes media y baja contaminan el agua con aguas residuales domésticas. Por último, algunos de los habitantes de los barrios legalizados, que tienen acueducto y alcantarillado, contaminan el agua con residuos sólidos después de actividades de reciclaje o cuando no logran depositar la basura a tiempo en los contenedores, ya que son pocos y se llenan rápido.

Posiblemente, los habitantes que contaminan el agua a través de diferentes usos y actividades son, en su mayoría, personas que se establecieron recientemente en los barrios de la microcuenca - "población flotante" - y que viven en arriendo, o se trata de nuevas generaciones que no tienen el mismo sentido de pertenencia que sus primeros habitantes y por eso no sienten la misma necesidad por cuidar el recurso hídrico y el ecosistema que en el pasado les permitió sobrevivir. Así se empiezan a generar los trade-offentre usuarios, donde los primeros pobladores son los perdedores con respecto a los otros pobladores mencionados, en relación con el SE de aprovisionamiento hídrico.

Otros trade-off entre usuarios dependen de su ubicación con respecto a la quebrada. Los que viven en la parte alta de la microcuenca serían los ganadores con respecto a los de las partes media y baja, debido a la reducción en la cantidad y calidad del agua a medida que va corriendo cuenca abajo. Esto sucede cuando se hace extracción directa del agua o se crean pozos y represas que retienen o desvían el curso natural del agua. Sin embargo, el problema más grande ocurre en el momento en que este recurso es transformado y depositado nuevamente a la quebrada, ya que su uso se vuelve el de vertedero de aguas residuales. Esta contaminación es acumulativa en tiempo y espacio, es decir, ya no hay agua de buena calidad para la mayor parte de los usuarios, a excepción de algunos ubicados en la parte alta de la quebrada, donde la actividad en ella es mínima y por tanto su afectación también.

Si bien para los actores las afectaciones pueden ser diferentes, a la larga el agua como recurso de uso común y la disminución de su calidad y cantidad terminan afectándolos a todos. Si viven cerca de la quebrada, pueden estar afectados por la contaminación, la generación y propagación de plagas y enfermedades relacionadas con malos olores que, además de molestos, pueden producir enfermedades respiratorias. Por otro lado pueden presentarse enfermedades causadas por morde- territarias 38

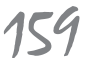


dura de roedores, los cuales son atraídos por las basuras presentes en la quebrada; además de alergias por picadura de insectos y enfermedades gastrointestinales, si se llega a consumir el agua de forma directa.

A escalas más amplias, los habitantes de esta microcuenca se sienten privilegiados con respecto a la mayoría de ciudadanos de Usaquén y de la ciudad de Bogotá, pues sienten que el hecho de vivir cerca de los cerros orientales y de tener una quebrada para observar les permite disfrutar muchos más servicios ecosistémicos. Por otro lado, la pérdida de cobertura vegetal disminuye otros SE, como la purificación del aire y el embellecimiento de la zona, que genera alegría en los habitantes. Sin embargo, a veces esta misma cobertura vegetal puede causar pérdida de calidad de vida, por cuanto se considera un escondite para delincuentes y consumidores de droga, y esto crea inseguridad en el sector, que los lleva a adoptar medidas como la eliminación de dichos árboles.

Los trade-offentre instituciones públicas y usuarios residentes urbanos aparecen cuando las primeras toman decisiones y ejercen acciones sobre el territorio, como la instalación del servicio de acueducto y alcantarillado en las viviendas legales, y el de acueducto en las viviendas ubicadas en la ronda de la quebrada. Con esta decisión se beneficiaron muchas personas que hacían largos desplazamientos hasta la quebrada para obtener agua, pero se perdió el interés por la quebrada y su conservación. Como resultado varias instituciones "pierden", como el caso de la Empresa de Acueducto y Alcantarillado de Bogotá, que es la institución encargada de la gestión del recurso hídrico; el hospital de Usaquén, que vela por la salud de las personas, y la Secretaría Distrital de Ambiente, que es la autoridad que promueve, orienta y regula la sustentabilidad ambiental de Bogotá.

Por otro lado, instituciones públicas y privadas, así como los usuarios residentes urbanos locales, reconocen que no es clara la situación legal en la que se encuentran los habitantes de la microcuenca, quienes están invadiendo el espacio denominado público, según la normativa colombiana, por habitar en viviendas construidas en ronda de la quebrada o en la reserva forestal. Lo que quiere decir que, aunque la ley esté escrita, los objetivos de conservar el agua y las áreas protegidas no se cumplen, pero sí reducen la calidad de vida de los habitantes cuando se toman decisiones que afectan su bienestar, como la declaración de sus predios como ilegales por parte de la Alcaldía Mayor de Bogotá. Aquí pierden los habitantes y pierden las instituciones como la Secretaría Distrital de Planeación y la Alcaldía Local, que se encargan de delimitar, vigilar y controlar lo que sucede en el borde urbano-rural de los cerros orientales de Bogotá.

Finalmente, las características actuales del ecosistema y el SE de aprovisionamiento hídrico han llegado a un estado donde las soluciones no pueden venir únicamente de la población que habita en la microcuenca, que a pesar del interés que manifiesta a través de las organizaciones comunales, no tiene el control sobre muchas de las 
decisiones. De igual manera, las intervenciones institucionales pueden llegar a ser insuficientes para solucionar las problemáticas donde prima el bienestar de la población local. En síntesis, el trabajo conjunto y coordinado entre los actores sociales relacionados con el manejo, el control y la vigilancia del recurso hídrico, en conceso con las necesidades de la población, pueden llegar a mitigar las pérdidas que se generen con las decisiones que se tomen sobre el SSE de la microcuenca de la quebrada San Cristóbal.

\section{Conclusiones}

El análisis de los trade-off temporales en el SSE de la microcuenca de la quebrada San Cristóbal permite ver los momentos críticos donde se tomaron decisiones que fueron definitivas en la transformación del ecosistema y en su capacidad de prestar el SE de aprovisionamiento hídrico. Esto genera una serie de lecciones que deben ser aprendidas para lograr una gestión exitosa a través de políticas y acciones que minimicen los efectos negativos de la expansión urbana hacia los cerros orientales de Bogotá. Los trade-off espaciales ayudan a comprender la complejidad del SSE ubicado en esta la franja urbano-rural, en la medida en que se reconoce su conexión a través de las diferentes escalas espaciales y se identifican los puntos o variables clave donde se debe intervenir para reducir los efectos negativos a escalas mayores y también en la escala de la microcuenca en sus distintos niveles. Los trade-off sociales resaltan la importancia de la participación de diferentes tipos de actores sociales que intervienen o hacen uso directo de los SE, y la incorporación de su visión en torno al problema contribuye a la caracterización de actores y procesos, e influencia las acciones sobre el recurso y el SE, y las perspectivas de acción.

La caracterización y el análisis de los actores e intereses que interactúan en diferentes escalas y que influencian, de diferentes maneras, la posibilidad de prestación de los servicios ecosistémicos en cuestión, aportan elementos para entender la complejidad del sistema socio ecológico sobre el que se busca tomar decisiones de gestión ajustadas a la situación. Este análisis deja ver esa complejidad y los retos que ella plantea, en la medida en que permite entender los límites de las decisiones que pueden tomar distintos actores en diferentes escalas, al igual que los resultados que se pueden obtener mediante las acciones posibles a desarrollar en cada una de ellas.

En este sentido es claro que, en el caso analizado, la recuperación o el mejoramiento del SE de provisión y calidad del agua ya ha llegado a un punto donde las acciones de conservación en la escala local contribuirían, en el mejor de los casos, a limitar un desgaste aun mayor. Por lo que se requieren, al mismo tiempo, decisiones y acciones de política en otros niveles, capaces de potenciar los logros a escala local.

Así mismo, la mitigación de las pérdidas que puedan generar las decisiones en torno al SSE de la microcuenca en distintos niveles exige la coordinación de los esfuer- territarias 38 
zos entre actores sociales e institucionales hacia un objetivo común de manejo, control, vigilancia para el mantenimiento de la calidad y cantidad del recurso hídrico, acorde con las necesidades de la población local y aquellas que tienen que ver con la generación del SE en escalas mayores.

\section{Referencias}

Acueducto. Agua y alcantarillado de Bogotá. (2003). Sistema hidrico de Bogotá. Recuperado de www.riosbogotá.org: https://docs.google.com/viewer?a= $\mathrm{v} \&$ pid=forums\&srcid=MTQxODM5 ODI3MDczMjAxNDA4NzEBMDE 5MDkyOTQwNTA2NTU5MTAzN zgBMkY4dXV4RmlEVmtKATQBAX Yy\&pli=1

Albán, M. (2007). La información disponible sobre los servicios de ecosistemas de montaña en los Andes del Norte y Centro. Recuperado de www.paramo. org: http://www.paramo.org/dvd/ Paramo\%20Andino\%20coordinación/ Componente\%204/4C.\%20Información\%20para\%20tomadores\%20de $\% 20$ decisión/IINFOR l.pdf

Alcaldía Mayor de Bogotá D. C. (1998). Decreto 1504. Recuperado de http:// www.alcaldiabogota.gov.co/sisjur/normas/Normal.jsp?i=1259

Alcaldía Mayor de Bogotá D. C. (2011). Decreto 575. Recuperado de http://www. alcaldiabogota.gov.co/sisjur/normas/ Normal.jsp?i=45006

\section{territarias 38} 162 de http://www.alcaldiabogota.gov.co/ sisjur/normas/Normal .jsp?i=57468\#0

Ataroff, M. \& Rada, F. (2000). Deforestation impact on water dynamics in a Venezuelan Andean cloud forest. AMBIO: A Journal of the Human Environment, 29(7), 440-444.

Bohórquez Alfonso, I. A. (2009). Observatorio rural bogotá. En La frontera rural de bogotá: un debate sobre sostenibilidad ambiental y crecimiento urbano. Recuperado de http://observatorioruralbogota.gov.co/apc-aa-files $/ 57 \mathrm{c}$ 59a889ca266ee6533c26f970cbl4a/ la_frontera_rural_de_bogota_un_debate_sobre_sostenibilidad.pdf.

Corporación Autónoma Regional de Cundinamarca - CAR (2009). Adecuación bidráulica y recuperación ambiental Rio Bogotá. Recuperado de http:// www.car.gov.co/recursos_user/Proyectos\%20Especiales/RIO\%20BOGOTA/Evaluacion\%20Ambiental\%20Volumen\%20III.pdf

Corredor Camargo, E. S., Fonseca Carreño, J. A. \& Páez Barón, E. M. (2012). Los sevicios ecosistémicos de regulación: tendencias e impacto en el bienestar humano. UNAD, 77.

Díaz, S., Quétier, F., Cáceres, D. M., Trainor, S. F., Pérez-Harguindeguy, N., Harte, B. et al. (2011). Linking functional diversity and social actor strategies in a framework for interdisciplinary analysis of nature's benefits to society. Proceedings of the National Academy of Sciences, 3, 895-902.

Elcy Corrales Roa, Viviana Osorno Acosta 
EAB, CAR \& CI (2009). Reserva forestal protectora Bosque Oriental de Bogotá inventario de fauna. Bogotá: Empresa de Acueducto y Alcantarillabo de Bogotá, Corporación Autónoma Regional de Cundinamarca y Conservación Internacional.

Ecosystem Services for Poverty Alleviation - ESPA (2012). Participatory modelling of wellbeing trade-offs in Coastal Kenya. Tools and processes for participatory modelling of wellbeing implications ecosystem service tradeoffs: description and reflection on methods developed and used in the ESPA P-Mowtick project. Recuperado de http://www.espa.ac.uk/projects/ ne-i00324x-1.

Empresa de Acueducto y Alcantarillado de Bogotá - EAAB, Corporación Autónoma Regional de Cundinamarca - CAR \& Conservación Internacional Colombia (CI) (2009). Reserva Forestal Protectora Bosque Oriental de Bogotá Inventario de Fauna. Bogotá: CAR y Conservación Internacional.

Etienne, M., Du Toit, D. \& Pollard, S. (2011). ARDI: A co-construction method for participatory modeling in natural resources management. Ecology and society, 16(1), 14.

Fallot, A. (2013). Guía metodologica PARDI: Problemática - Actores - Recursos - Dinámicas - Interacciones para el análisis de las dinámicas socio-ecológicas. Recuperado de http://hal.cirad.fr/file/index/ docid/933599/filename/2013_08_ Fallot_Metodologia_PARDI.pdf
Galvis, L. A. (2013). ¿El triunfo de Bogotá?: desempeño reciente de la ciudad capital. Coyuntura económica: investigación económica y social, XLIII(1), 199-236.

Garzón Díaz, F. A. (2014). Educación ambiental y desarrollo sostenible. El caso de los cerros Orientales de Bogotá, Colombia. Revista latinoamericana de bioética, 14(1), 82-97.

Hospital de Usaquén (2006). Reconstrucción de la memoria colectiva en salud. Grupo "Los Conquistadores”. Barrio Cerro Norte. Bogotá: Alcaldía Mayor de Bogotá D.C.

Hospital de Usaquén (2012). Diagnóstico local con participación social 2010-2012. Localidad 01 de Usaquén. Recuperado de hospitalusaquen.gov.co

IDIGER, Fundación Manigua (junio de 2015). Quebrada San Cristóbal. Recuperado de http:/ /quebradasancritobal.jimdo. com/inicio/convenio-506/

Martín-López, B., \& Montes, C. (2011). Biodiversidad y servicios ecosistémicos ante el cambio global. En Biodiversidad en España: base de la sostenibilidad ante el cambio global (pp. 444-465). Madrid: Observatorio de la Sostenibilidad en Españal.

Martín-López, B. \& Montes, C. (2010). Funciones y servicios de los ecosistemas: una herramienta para la gestión de los espacios naturales. Guía cientifica de Urdaibai, 1, 13-32.

Martín-López, B., García-Llorente, M., Gómez-Baggethun, E. \& Montes, C. (2010). Evaluación de los servicios de los ecosistemas del sistema socio-eco- 
lógico de Doñana. (Catedra-UNesco, Ed.). Revista de la Cátedra Unesco sobre desarrollo sostenible de la UPV/EHU, (4), 91-111.

Martín-López, B., González, J. A. \& Vilardy, S. (2012). Guia docente ciencias de la sostenibilidad. Bogotá: EdiPrint Ltda.

Mathevet, R., Etienne, M. \& Lynam, T. (2011). Water management in the Camargue Biosphere Reserve: insights from comparative mental models analysis. Ecology and society, 16(1), 20.

Observatorio Nacional de Paz (2009). "Cerro norte": conflictos y propuestas de transformación social, territorial y ambiental hacia la paz en Bogotá. En Documentos mensuales de análisis y divulgación sobre conflictos, Región Centro. Bogotá: Observapaz.

Reina Rozo, J. (2013). Metabolismo Social: Hacia la sustentabilidad de las transiciones socioecológicas urbanas. Bogotá: Universidad Nacional de Colombia.

Secretaría Distrital de Ambiente - SDA \& Conservación Internacional - CI (2010). Politica para la gestión de la conservación de la biodiversidad en el Distrito Capital. Bogotá: Panamericana.

Secretaría Distrital de Ambiente - SDA (2009). Agenda ambiental localidad de Usaquén. Recuperado de http://riosalitre. files.wordpress.com/2009/04/1_aa_ usaquen2.pdf

Secretaría Distrital de Ambiente- SDA, Fondo de Desarrollo Local de Usaquén \& Conservación Internacional Colombia (2014). Las quebradas de Usaquén, reconciliarse con las Quebradas. Recupe- rado de https://quebradasusaquen. wordpress.com/proyecto-quebradas / Secretaría Distrital de Planeación \& Secretaría de Hacienda Distrital (2004). Recorriendo Usaquén: Diagnóstico de los aspectos físicos, demográficos y socioeconómicos. Recuperado de impuestos. shd.gov.co: impuestos.shd.gov.co/.../ localidades.../RECORRIENDO_USAQUEN.pdf

Secretaría Distrital de Planeación - SDP (2007). Los caminos de los cerros. Bogotá: Alcaldía Mayor de Bogotá, Dirección de Ambiente y Ruralidad.

Secretaría Distrital de Planeación - SDP (2009). Conociendo la localidad de Usaquén: Diagnóstico de los aspectos fisicos, demográficos y socioeconómicos. Bogotá: Alcaldía Mayor de Bogotá.

Secretaría Distrital de Planeación - SDP (2015). Resolución 228 del 2015. Recuperado de http://static.iris.net.co/ sostenibilidad/upload/documents/ Documento_32690_20150311.pdf

Secretariat of the Convention on Biological Diversity - SCBD (2012). Cities and biodiversity out. Montreal: Stockholm Resilience Center.

Tobón, C. (2009). Los bosques andinos y el agua. Programa regional ECOBONA -Intercooperation Ed. (P. M. Caracola, Ed.). Quito: Serie investigación y sistematización, n. ${ }^{\circ} 4$.

Universidad El Bosque (2013). Aportes para la ordenación del planeamiento del uso y manejo sostenible de los recursos hidricos de agua dulce en las microcuencas de la localidad de Usaquén, Distrito Capital.

Elcy Corrales Roa, Viviana Osorno Acosta 
CÓDIGO DI: PCI 2011-230. Bogotá D. C.

Universidad El Bosque (2014). Caracterización y evaluación de la calidad biólogica y fisicoquimica del agua de la microcuenca de San Cristóbal. Universidad El Bosque, Ingeniería Ambiental, Bogotá.
Van Der Hammen, T. (1998). Plan ambiental de la Cuenca Alta del río Bogotá (análisis y orientaciones para el ordenamiento territorial). Bogotá: Corporación Autónoma Regional de Cundinamarca, CAR. 
